\title{
HD 34700 is a T Tauri multiple system
}

\author{
M. F. Sterzik ${ }^{1}$, C. H. F. Melo ${ }^{1}$, A. A. Tokovinin ${ }^{2}$, and N. van der Bliek ${ }^{2}$ \\ ${ }^{1}$ European Southern Observatory, Casilla 19001, Santiago 19, Chile \\ e-mail: msterzik@eso.org \\ ${ }^{2}$ Cerro Tololo Inter-American Observatory, Casilla 603, La Serena, Chile
}

Received 2 November 2004 / Accepted 28 December 2004

\begin{abstract}
We have imaged the young double-lined spectroscopic binary HD 34700 in the near-infrared and find evidence for two faint, visual, stellar components at 5'.2 and 9.'2 distance. High-resolution echelle spectroscopy of both stars shows strong Li I $6708 \AA$ absorption and $\mathrm{H} \alpha$ emission. The spectral types of the companions are estimated from the spectra and photometry as M1-M2 and M2-M3. Their radial velocities are similar to the center-of-mass velocity of the central SB2; hence all four stars are most probably physically bound and constitute a young quadruple stellar system with an inner short-period binary. We provide a list of pre-main sequence spectroscopic binaries with additional components of which HD 34700 is yet another example. The available statistics strengthen the suspicion that dynamical effects in multiple systems play a key role in the formation of very close binaries early in their evolution.
\end{abstract}

Key words. binaries: spectroscopic - stars: formation - binaries: close - binaries: general - stars: pre-main sequence

\section{Introduction}

The star HD 34700 (HIP 24855) has recently been identified as a new double-lined spectroscopic binary system by Arellano Ferro \& Giridhar (AFG, 2003). Their analysis shows that both components of the SB2 have strong Li I $6708 \AA$ absorption and variable $\mathrm{H} \alpha$ emission lines, thus justifying classification as a pair of $\mathrm{T}$ Tauri stars. Both components have an approximate spectral type of G0 IVe (Mora et al. 2001), and associated strong infrared excess has been known since IRAS (Oudmaijer et al. 1992). The spectral energy distribution can be interpreted by a disk model (Sylvester \& Skinner 1996), and the presence of circumstellar material is further supported by the detection of ${ }^{12} \mathrm{CO}$ and ${ }^{13} \mathrm{CO}$ emission (Zuckerman et al. 1995). The star is also a strong X-ray source, and given its location in the general direction towards Orion, its relative youth is undisputed. However, a more quanititative age determination is difficult, mainly because its parallax is rather uncertain in $\operatorname{HIPPARCOS}\left(\pi_{\mathrm{HIP}}=0.86 \pm 1.84\right.$ mas $)$.

The number of known double-lined pre-main-sequence (PMS) spectroscopic binaries is still small (Melo et al. 2001). Motivated by general interest studying them in depth, Torres (2004) presents a series of high-resolution spectra of HD 34700. He confirms its youth, solves an accurate orbit with a period of 23.4877 days and an eccentricity of 0.2501 , and finds that the stars are not tidally synchronized, because the projected rotational velocities measured are much larger.

Our own interest in this stellar system was raised when looking at the 2MASS images of HD 34700. The explanation given in AFG (their Fig. 5) is somewhat ambiguous and mentions asymmetric emission, either caused by dust or by an unresolved star. However, different cuts used to display the $2 M A S S J H K$ images already start to resolve the system into three components. In fact, all three are listed in the $2 M A S S$ point-source catalog (Cutri et al. 2003).

The goal of our work was to unambiguously resolve the individual stars and to look for a potential physical relation of the wide components with the central SB2. We present new near-infrared (NIR) images and optical high-resolution spectroscopy of all stars which allow us to derive radial velocities, spectral types, and the Li I strength as youth indicator. We show that our data are consistent with the notion that HD 34700 is in fact a young quadruple system, consisting of an inner SB2 and two late-type companions in wide orbits.

We compare the properties of this new system of T Tauri stars with the growing list of multiple, low-mass, pre-main sequence stars in (mostly) hierarchical configurations. HD 34700 might be another example of a stellar system in which the inner, close binary is a result of dynamical interactions in multiple systems early in their evolution.

\section{Imaging}

HD 34700 was imaged on January 30, 2004, using the Infrared Side-Port Imager (ISPI) mounted at the CTIO $4 \mathrm{~m}$-Blanco telescope (Probst et al. 2003). $J H K$ and narrow-band $\mathrm{Br}-\gamma$ images were secured under photometric conditions. A pixel scale of 0.306 and sub-arc second image quality ensure that three visual components, called hereafter $\mathrm{A}, \mathrm{B}$, and $\mathrm{C}$, are well resolved (Fig. 1). 


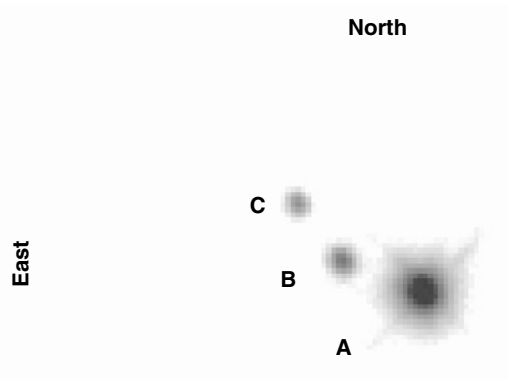

Fig. 1. $K$-band image of HD 34700 observed with ISPI. North is up, and east is to the left. Separations, postion angles, and photometry are given in Table 1.

Table 1. Results of IR imaging for HD 34700.

\begin{tabular}{lllllll}
\hline \hline & Sep. ["] & PA [ $\left.{ }^{\circ}\right]$ & $J$ & $H$ & $K$ & $\mathrm{Br} \gamma^{a}$ \\
\hline A & - & - & - & - & $7.7^{b}$ & 0.0 \\
B & $5.18 \pm 0.01$ & $69.1 \pm 0.1$ & $12.29^{c}$ & $11.52^{c}$ & $11.03^{c}$ & 3.3 \\
C & $9.17 \pm 0.01$ & $54.9 \pm 0.1$ & $12.78^{c}$ & $12.14^{c}$ & $11.91^{c}$ & 4.2 \\
\hline
\end{tabular}

${ }^{a}$ Instrumental magnitudes.

${ }^{b}$ Extrapolated from the relative $\mathrm{Br} \gamma$ magnitudes of $\mathrm{A}, \mathrm{B}$, and $\mathrm{C}$. Its error is $0.1^{\mathrm{m}}$.

${ }^{c}$ Errors in the photometry of components B and C is $0.03^{\mathrm{m}}$.

We derive the relative astrometry and photometry from PSF fitting, using 6 nearby stars from the $2 M A S S$ point-source catalog (Cutri et al. 2003) as standards. Unfortunately, component A is slightly saturated in the broadband images, compromising its photometry. We estimate the errors of the photometry of the components $\mathrm{B}$ and $\mathrm{C}$ in the $J H K$ images to be less than $0.03^{\mathrm{m}}$, while errors in the $\mathrm{Br} \gamma$ band are higher because of the faintness of the sources. Nevertheless, the $\mathrm{Br} \gamma$ relative magnitudes between $\mathrm{A}, \mathrm{B}$ and $\mathrm{C}$ are a useful approximation for the broad-band $K$ magnitudes, and we derive a $K \approx 7.7 \pm 0.1$ for component $\mathrm{A}$. This value is also consistent with the 2MASS combined $K$-band magnitude of 7.48 for the system. Results of the astrometry and photometry are summarized in Table 1 . The consistency of the relative astrometry between several images is about $0{ }^{\prime} 01$.

We note that the NIR colors of the component $\mathrm{C}$ match those of a dwarf with a spectral type between M2 and M3. The $H-K \approx 0.5$ color of component B indicates its substantial reddening. We tentatively conclude that this component is most likely affected by circumstellar dust absorption, an interpretation fully consistent with observed broad and variable $\mathrm{H}_{\alpha}$ profiles (see below).

Interestingly, HD 34700 was observed with NICMOS in coronographic mode onboard HST already on September 17, 1998. Two short $(0.826 \mathrm{~s})$ acquisition images in the filter F165M easily resolve the system into three point sources. The astrometry based on calibrated frames accessible via the public archive yields a separation of $5^{\prime \prime} \cdot 23$ and $\mathrm{PA}=69.3^{\circ}$ between components $\mathrm{A}$ and $\mathrm{B}$ and a separation of 9!'25 and $\mathrm{PA}=$ $55.1^{\circ}$ between $\mathrm{A}$ and $\mathrm{C}$. These values are thus fully consistent with our 2004 epoch observations. However, the small proper motion of HD 34700 of $\sim 1$ mas/yr does not allow the conclusion that this is a common proper motion system over a short baseline of 6 years.

\section{Spectroscopy}

Guided by the accurate astrometry of the ISPI image, we performed spectroscopy of each of the three visual components of HD 34700 on March 4, 2004 under photometric conditions. We used the Fibre-fed Extended Range Optical Spectrograph (FEROS) attached to the ESO $2.2 \mathrm{~m}$ telescope in La Silla, offering a spectral resolution of 48000 and a coverage between $\sim 3500 \AA$ and $\sim 9200 \AA$ distributed over 32 echelle orders (Kaufer et al. 1999). Centering of components B and C was achieved by issuing relative offsets with respect to the primary SB2. Although all three components were fully resolved in the acquisition camera under seeing conditions of $0 !^{\prime} 9$, the central SB2 saturates the images, and telescope co-guiding on an anonymous field star was performed instead. The fiber of FEROS has a diameter of $2{ }^{\prime \prime} 0$, and component B is located about 5'.0 from the central source. Assuming a Gaussian profile of $1{ }^{\prime \prime} 0$ width for the central source, we estimate the flux contamination at the location of component $\mathrm{B}$ to be negligible. Some low-level contamination, however, is found after cross-correlation (see below). For reference, the spectrum of the bright central SB2 was recorded, too. The integration time was $10 \mathrm{~min}$ for the SB2, and 20 min for each of the components $\mathrm{B}$ and $\mathrm{C}$. The data reduction was performed in a standard way using the FEROS pipeline (bias, background correction, flat-fielding, optimal extraction and wavelength calibration). While the spectrum of A exhibits a very high $S / N$ of $\sim 150$ at $5600 \AA$ (as expected for an object of $V=9.15$ ), the spectra of B and C are noisy with $S / N$ of $\sim 7$ and $\sim 3$, respectively.

We measure approximate flux ratios of $f_{\mathrm{B} / \mathrm{A}}=0.0017$ and $f_{\mathrm{C} / \mathrm{A}}=0.0010$ in the continuum around $5600 \AA$ ( $V$ band $)$. This means that $\mathrm{B}$ and $\mathrm{C}$ are roughly 6.9 and $7.5 \mathrm{mag}$ fainter than the primary, respectively. Assuming the visual apparent magnitude of the A component to be $V_{\mathrm{A}}=9.15$, we estimate $V_{\mathrm{B}} \sim 16$ and $V_{\mathrm{C}} \sim 16.5$. We caution that these are only indicative for the true optical magnitudes. Spectrophotometric flux calibration is unreliable with these fibre-fed observations due to variations in seeing and object centering, as well as the low $S / N$.

\subsection{Spectral types}

Due to the low signal-to-noise, spectral types for B and C components can only be determined roughly. An estimate can be obtained by comparing the absolute visual magnitudes with those predicted by evolutionary models. Following Torres (2004), we may assume that HD 34700 is actually located in Orion (i.e. at a distance of $d=450 \mathrm{pc}$, corresponding to a distance modulus of $8.2 \mathrm{mag}$ ) with an age of 3 Myrs. Referring to Baraffe et al. (1998), we translate the infrared magnitudes into a mass of $\sim 0.6 M_{\odot}$ and $T_{\text {eff }} \sim 3650$ for B, 
$\sim 0.4 M_{\odot}$ and $T_{\text {eff }} \sim 3450$ for $\mathrm{C}$. We note, however, that component $\mathrm{B}$ is highly reddened and does not allow a reliable classification. Using the effective temperature-spectral type relation given for class IV stars (de Jager \& Nieuwenhuijzen 1987) $\mathrm{B}$ and $\mathrm{C}$ should then have spectral types around M1 and M2, respectively ${ }^{1}$.

Visual comparisons of the spectra for the B and C components with the spectral library of Montes et al. (1997) fully agree with this estimate and give spectral types between M1-2 and M3-4.

\subsection{Youth indicators}

Since Li is strongly depleted in the interior of late-type fullyconvective stars already during the pre-main sequence phase, the presence a strong Li I absorption in the atmosphere of these low-mass stars is a strong youth indicator.

AFG and Torres (2004) tried to assess the age of the SB2 with $\mathrm{Li}$ I, too. However, due to the G0 spectral types, the Li criterion does not pose strong youth constraints. In Fig. 2, we show a close-up of the spectral region around the Li I $6708 \AA$ for all three components. The A component is shifted to the rest frame using the systemic velocity given by Torres (2004) whereas, for the $\mathrm{B}$ and $\mathrm{C}$ components, we use the radial velocities computed from our cross-correlation functions (see Table 2). Although the spectra of components B and C are much noisier than A, they still allow us to determine key quantities of the stars. Strong Li I absorption is clearly evident for all objects. For each component of $\mathrm{A}$ we measure a lithium equivalent width of $0.15 \AA$, compatible with the $0.17 \AA$ reported by Torres (2004). The Li I absorption lines for B and C fall nicely in between the well-separated components of the SB2, which already indicates that the radial velocities of $\mathrm{B}$ and $\mathrm{C}$ are close to the center-of-mass velocity of the SB2 system. The Li I equivalent widths of $\mathrm{B}$ and $\mathrm{C}$ are much higher; we measure $0.5 \AA$ and $0.7 \AA$ but assign somewhat larger error bars $(0.2 \AA)$ due to the noise in the line and continuum.

According to Martin (1997), all stars with spectral types later than $\mathrm{K} 7$ which have $\mathrm{Li} \mathrm{I}$ absorption lines with equivalent widths larger than $0.55 \AA$ are genuine T-Tauri stars. We conclude that the two late-type companions are therefore bonafide TTS. Due to high uncertainties in the equivalent width and in the spectral types we do not attempt to estimate their Li abundances more quantitatively. But we note that in the diagram of Li equivalent width versus temperature both stars are indistinguishable from the pre-main sequence TTS population identified in Orion (Alcalá et al. 2000) and Taurus-Auriga (Wichmann et al. 2000) and aged in the range from 1 to $10 \mathrm{Myr}$.

Another common youth indicator for TTS is the $\mathrm{H}_{\alpha}$ line which appears in emission as a result of chromospheric activity and/or accretion of circumstellar material. A close-up around the $\mathrm{H}_{\alpha}$ region is shown in Fig. 3. In the SB2 (top), $\mathrm{H} \alpha$

1 Torres (2004) alternatively estimates a distance of $250 \mathrm{pc}$ and age of about $9 \mathrm{Myr}$ for the SB2. Unfortunately, the errors of our measured colors for $\mathrm{C}$ do not allow us to distinguish between both options, as the colors and magnitudes are compatible with both possibilities of distance/age combinations.

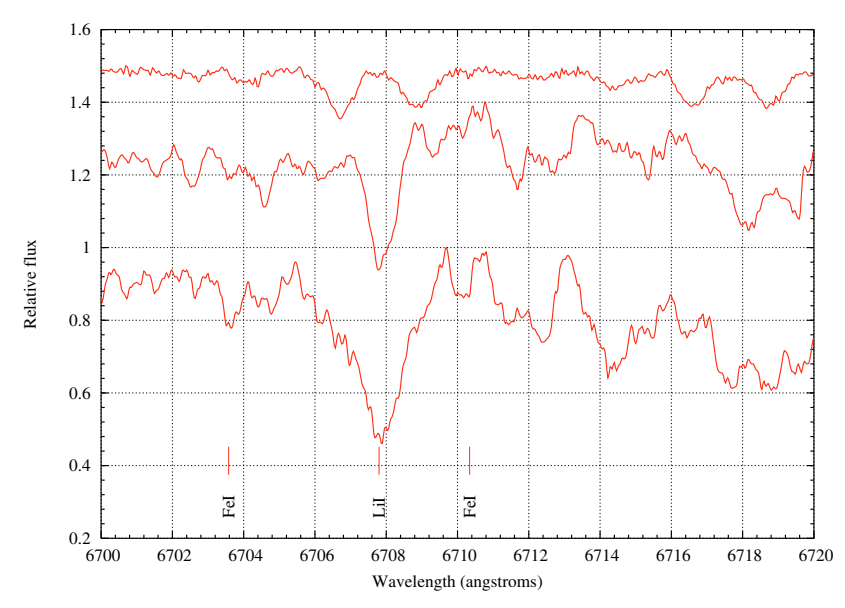

Fig. 2. High-resolution spectra of all components of HD 34700 in the Lithium region (top to bottom: A, B, C).

Table 2. Spectroscopic results for HD 34700: equivalent widths, radial velocities $V_{\mathrm{r}}$, and rotation $V \sin i$. Observations are performed on $\mathrm{JD}=2453069.522$.

\begin{tabular}{lllll}
\hline \hline Source & $\begin{array}{l}\mathrm{Li} \mathrm{I}(6708) \\
\AA\end{array}$ & $\begin{array}{l}\mathrm{H} \alpha(6562) \\
\AA\end{array}$ & $\begin{array}{l}V_{\mathrm{r}} \\
\mathrm{km} \mathrm{s}^{-1}\end{array}$ & $\begin{array}{l}V \sin i \\
\mathrm{~km} \mathrm{~s}^{-1}\end{array}$ \\
\hline $\mathrm{Aa}$ & $0.15 \pm 0.05$ & - & $68.04 \pm 0.2$ & $26.3 \pm 0.6$ \\
$\mathrm{Ab}$ & $0.15 \pm 0.05$ & - & $-27.98 \pm 0.2$ & $21.7 \pm 0.6$ \\
$\mathrm{~B}$ & $0.5 \pm 0.2$ & $25 \pm 2$ & $20.13 \pm 0.4$ & $11.5 \pm 1.0$ \\
$\mathrm{C}$ & $0.7 \pm 0.2$ & $6 \pm 2$ & $18.69 \pm 1.5$ & $27.5 \pm 1.7$ \\
\hline
\end{tabular}

has a complicated and broad profile, as already noted by AFG and Torres (2004). Instead, both components B and $\mathrm{C}$ show a broad and strong $\mathrm{H}_{\alpha}$ in emission with equivalent widths of $25 \AA$ and $6 \AA$, respectively. B is formally classified as classical TTS and $\mathrm{C}$ as weak-line TTS. In particular, as discussed in Edwards et al. (1994), the broad $\mathrm{H}_{\alpha}$ profile in emission, a blue shifted component in absorption and $H-K$ excess, all suggest a circumstellar accretion disk around B. This is especially interesting as HD 34700 then represents an example of the relatively rare binary or multiple systems in which stars with and without noticeable circumstellar material are mixed (Prato et al. 2003).

\subsection{Radial velocities and $V \sin i$}

Radial velocities have been derived by cross-correlating the observed spectra with a numerical mask, resulting in a crosscorrelation function (CCF). Details of the cross-correlation procedure are given in Melo et al. (2001). Cross-correlation has been performed using 4 different numerical spectral templates, namely, M4, K0, G2, F0. In the case of the A component, the intensity of the cross-correlation increased towards masks of earlier spectral types reaching its maximum for the G2 and decreasing again for the F0 mask, which is in agreement with the spectral types derived by AFG and Torres (2004). As an additional check, our radial velocities for both components of the SB2 can be compared to the radial velocities predicted by the ephemeris computed based on the orbital 


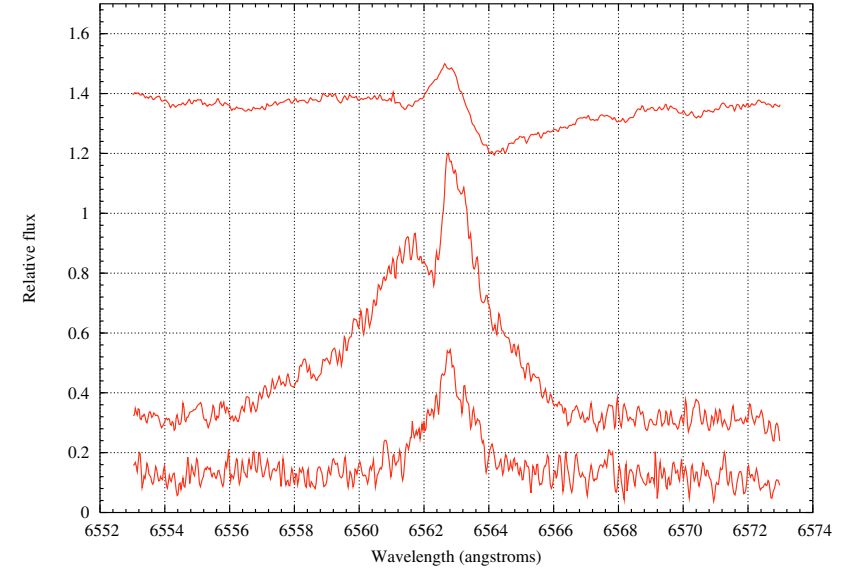

Fig. 3. High-resolution spectra of all components of HD 34700 in the $\mathrm{H} \alpha$ region (top to bottom: A, B, C).

elements given by Torres (2004). For the time of our observations, $\mathrm{JD}=2453069.522$ (i.e., $\phi=0.0217 \pm 0.008$ ), the predicted values are $68.8 \pm 1 \mathrm{~km} \mathrm{~s}^{-1}$ for the primary and $-27.3 \pm 1 \mathrm{~km} \mathrm{~s}^{-1}$ for the secondary, in full agreement with the values given in Table 2. For the $\mathrm{B}$ and $\mathrm{C}$ components, only the M4 mask produces meaningful cross-correlation peaks, which confirms the late-type spectral estimate above. The CCF computed with the K-F masks indicates a signal corresponding to the radial velocity of the SB2 and, therefore, some contamination. The CCF for all three components are shown in Fig. 4. For the A component (top) only the CCF produced with the $\mathrm{G} 2$ mask is shown, whereas for the $\mathrm{B}$ and $\mathrm{C}$ components an M4 mask was used. Since no simultaneous calibration lamp was used, radial velocity errors are dominated by the spectrograph shifts due to changes in ambient conditions (i.e., temperature and pressure). These shifts are typically about 100 $200 \mathrm{~m} / \mathrm{s}$ per night (for a discussion of the error budget, see Melo et al. 2001).

Projected rotational velocities $V \sin i$ were computed using the $\sigma(\mathrm{CCF})-V \sin i$ calibration for FEROS described in Melo et al. (2001). Our value for the A component is in very good agreement with those from Torres (2004). Unfortunately, no FEROS calibration exists for the M4 mask where the CCF for the $\mathrm{B}$ and $\mathrm{C}$ components has been computed. We have therefore utilized calibrations derived by Delfosse et al. (1998) for the ELODIE spectrograph with a similar resolving power (42 000). Thus, we believe that the $V \sin i$ given for the B and C components are reasonable estimates with an error of $1-2 \mathrm{~km} \mathrm{~s}^{-1}$.

\section{Discussion}

We find that the radial velocities of components B and C are, within the errors, consistent with the center-of-mass velocity of the central SB2. Moreover, these radial velocities are also consistent with radial velocities of TTS in the general direction of Orion. Alcalá et al. (2000) identified about 100 TTS scattered all around Orion. The most adjacent TTS to HD 34700 is RX J0519.9+0552, a K7 wTTS at a projected distance of $\sim 30^{\prime}$. Its radial velocity, however, differs from that ofHD 34700.
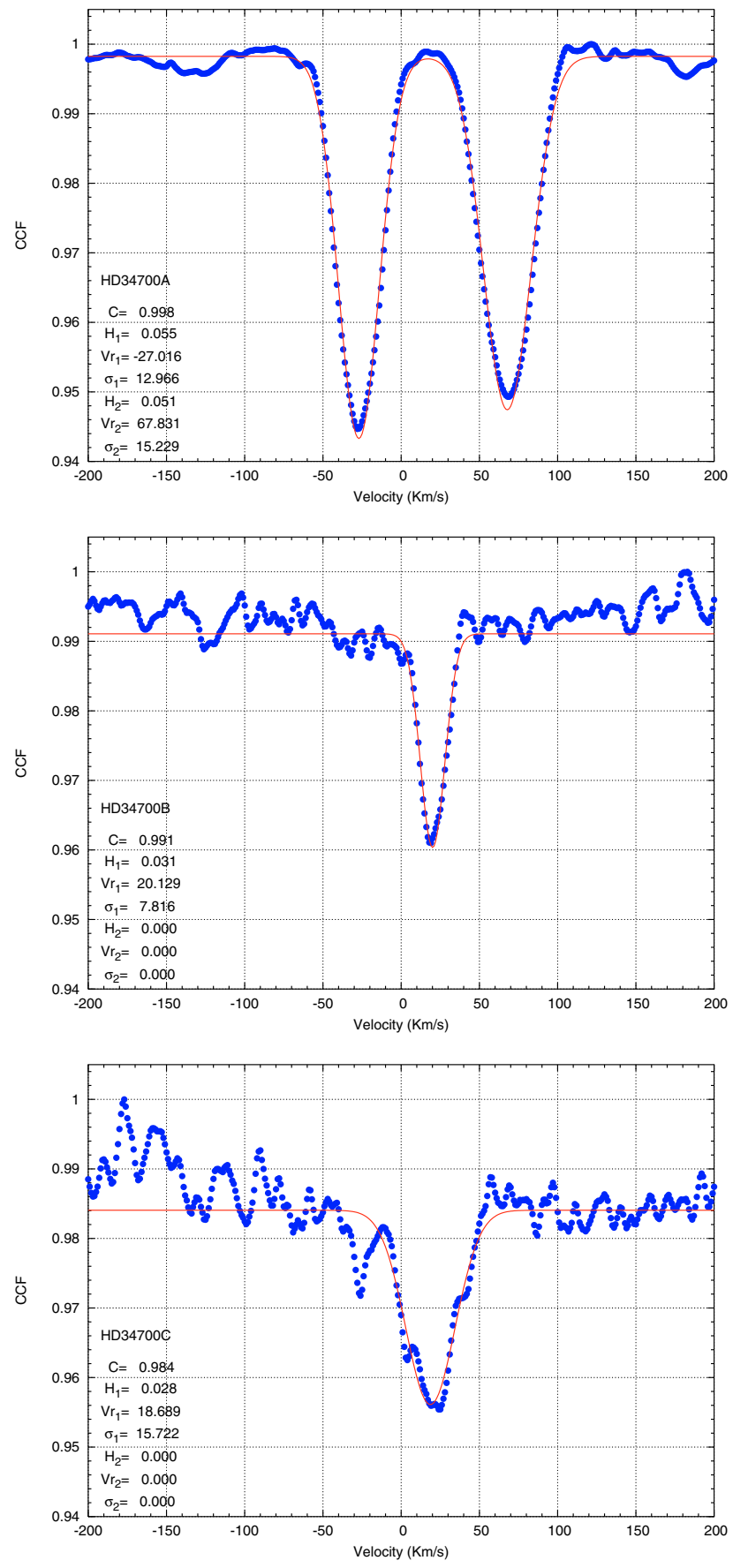

Fig. 4. Cross-correlation functions (top to bottom: A, B, C).

No prominent past or present star-forming clouds lie in the vicinity of these stars. The space density of TTS in this region being low, a chance projection of a physically unrelated TTS within an annulus of $10^{\prime \prime}$ around a given TTS is highly improbable (but not impossible). To find two chance projections in the same annulus appears even more implausible.

Considering the similar evolutionary state of all components, we tentatively conclude that HD 34700 forms a multiple TTS system consisting of an inner SB2 and two outer physical companions. We cannot make conclusive statements about the long-term dynamical stability of HD 34700 . The projected configuration of this quadruple appears non-hierarchical. Because 
Table 3. Multiple TTSs with SBs having a known orbit. The periods of spectroscopic binary $P_{\text {in }}$ (in days) and the angular distances (or periods) of outer components are listed.

\begin{tabular}{|c|c|c|c|}
\hline Source & $P_{\text {in }}$ & $d_{\text {out }}$ & Remark \\
\hline HD 155555 & 1.7 & $33^{\prime \prime}$ & \\
\hline V1154 Sco & 2.4 & 0.288 & \\
\hline RW Aur & 2.77 & $0 . ' 12+11^{\prime \prime} 39$ & Quad. \\
\hline RX J0529.4+0041 & 3.03 & $1 . .3$ & eclips. \\
\hline RX J0541.4-0324 & 4.98 & & SB3 \\
\hline RX J1301.1-7654 & 13 & 1." 44 & \\
\hline UZ Tau & 19.1 & $0.368+3^{\prime \prime} .54$ & Quad. \\
\hline HD 34700 & 23.5 & $5^{\prime \prime}+10^{\prime \prime}$ & Quad. \\
\hline ROXs 42C & 36 & 0.157 & \\
\hline RX J0532.1-0732 & 46.9 & & SB3 \\
\hline V773 & 51.1 & $0 . .2+0.2$ & Trap. \\
\hline Crux-3 & 58.3 & $4.6 \mathrm{yrs}$ & SB3 \\
\hline ROXs 43A & 89.1 & $6^{\prime \prime}$ & \\
\hline HD 98800 & $262+315$ & $0 .{ }^{\prime} 8$ & Quad. \\
\hline Haro 1-14 & 591 & 12.9 & \\
\hline
\end{tabular}

the projections of $\mathrm{B}$ and $\mathrm{C}$ are not known, their actual distances from A can be much larger. In fact, a factor of 4-5 larger distances make the system stable. On the other hand, at a distance of $450 \mathrm{pc}$ the observed separations of B and C imply a crossing time of the order of $10^{5} \mathrm{yr}$; hence even a nonhierarchical multiple system could survive during several Myr.

Interestingly, similar configurations are encountered quite frequently in TTS systems. In order to be able to make statistically meaningful comparisons, we restrict the following discussion to low-mass TTS systems where the spectroscopic orbit has been solved sufficiently accurately, and in which all components have spectral types later than F5. A fairly complete compilation of periods and eccentricities is given by Melo et al. (2001), and 37 PMS SBs follow our selection criterion. Together with two orbits of weak-line TTS SBs (RX J0528.0+1046 and RX J0529.3+1210) from Torres et al. (2002), and the classical TTS system RW Aur (Gahm et al. 1999) the number of currently known TTS systems that contain (at least) one SB with a solved orbit is 40 . Twelve of them have inner periods $P_{\text {in }}<10 \mathrm{~d}$.

In order to derive the fraction of multiple systems $(N>2)$, we have searched the literature and the Multiple Star Catalog (MSC, Tokovinin 1997) for all known TTS SBs with at least one additional component. We are not aware of any particular survey that has attempted to reveal visual companions specifically around TTS SBs, and the list of companions associated to an SB in Table 3 is likely to be incomplete.

Table 3 lists 15 systems that have at least one additional companion. Three of those are components inferred from high-resolution spectroscopy, i.e. are SB3. Seven are visual components from a hierarchical tertiary configuration (with separations ranging from 0.16 to $33^{\prime \prime}$ ), and the remaining 5 systems are quadruple. In our well-defined sample of PMSSBs, we thus determine the fraction of multiple systems (i.e. systems which are at least triple) to be $15 / 40=0.38 \pm 0.10$. Triple systems have a fraction of $10 / 40=0.25 \pm 0.08$, and the fraction of quadruples with respect to triples is $5 / 10=0.5 \pm$ 0.22 . If we restrict the sample to SB with inner periods $P_{\text {in }}<$ $10 \mathrm{~d}$, we find 4 triples and 1 quadruple, i.e. a higher-order multiplicity fraction of $5 / 12=0.42 \pm 0.19$.

We will now compare the incidence of additional companions to SBs in our sample with samples of SBs in the field and in clusters. In young star clusters the ratio of triples to binaries is consistently found to be around $10 \%$, and has been determined in the Pleiades (Mermilliod et al. 1992) and in Praesepe (Mermilliod \& Mayor 1999). Mayor \& Mazeh (1987) estimate that some $25 \%$ of SBs are triple. The fraction of triples increases when we restrict the discussion to close SBs only. All 5 systems with $P_{\text {in }}<10 \mathrm{~d}$ in Duquennoy \& Mayor (1991) are triple, and at least $43 \%$ of the nearby, low-mass SBs with $P_{\text {in }}<10$ d catalogued by Batten et al. (1989) have known physical tertiaries. The higher-order multiplicity fraction $(42 \%)$ in our PMS sample restricted to $P_{\text {in }}<10 \mathrm{~d}$ is therefore compatible with these numbers, if not lower.

We conclude that the frequencies of triples and quadruples in our entire sample of PMS SBs $(0.38 \pm 0.1)$ shows a slight trend to be enhanced as compared to field $(0.25)$ or young clusters stars (0.1), although the errors are still too large to draw unambiguous conclusions. For sub-samples restricted to close SBs, however, we find a similar multiplicity fraction.

Two observational effects are known that are compatible with the high abundance of higher-order systems in our sample. First it is established that the binary fraction of TTS is - at least in some star forming regions - significantly higher than in the field (Leinert et al. 1997; Ghez et al. 1997). The trend toward a higher multiplicity fraction in our sample may therefore simply reflect the overall tendency of observing higher multiplicities in earlier evolutionary phases, although the stars of our sample are well mixed throughout the sky and should not be dominated by regional selection biases.

The second observational fact is that the fraction of SBs in components of visual multiple stars has been found to be higher than those in stars without any companion. Melo (2003) finds an excess of 2-3 in the rate of PMS-SBs that have one or more visual companions, compared to those without a companion. A similar conclusion is reached in an analysis of the frequency of spectroscopic sub-systems among the components of visual binaries in the field (Tokovinin \& Smekhov 2002).

The statistics presented above therefore corroborate Melo's (2003) results and suggest that the multiplicity fraction of PMS-SBs is genuinely enhanced. A possible explanation is that the formation of a close binary is linked to the presence of a more remote companion. The distant companion can assist in the removal of the angular momentum from the close binary and the eventual shrinkage of its orbit by several different mechanisms, e.g. by Kozai cycles in combination with tidal dissipation (Kiseleva et al. 1998). We might therefore also understand the relative underabundance of multiples with small $P_{\text {in }}$ in our PMS sample compared to the field in the sense that secular processes are still acting to evolve the systems into more hierarchical and stable configurations. 
We also note that the decay of young multiple systems formed in few-body clusters is yet another possible scenario compatible with this notion (Sterzik \& Tokovinin 2001; Sterzik et al. 2003).

\section{Summary}

We have resolved HD 34700, a T Tauri double-lined spectroscopic binary system, by NIR imaging and found two additional faint, visual, stellar components at 5'.2 and 9.'2 distance from the primary system. Both stars are of late spectral type that show strong Li I absorption indicative of the PMS nature. Their radial velocities are consistent with the center-of-mass velocity of the primary, so we conclude that HD 34700 is most probably a physically bound multiple TTS system consisting of an inner SB2 and two late-type companions in wide orbits. At least one of the wider components is actively accreting.

The statistics of TTS with known spectroscopic orbits reveal that the fraction of multiple TTS that contain one close (SB) sub-system is enhanced as compared to the field. This strengthens the notion that the formation of close binaries is linked to the presence of a third stellar component and to dynamical interactions in multiple systems early in their evolution.

Acknowledgements. We acknowledge the comments of the referee, T. Mazeh, that helped to improve and clarify the manuscript, which is based on observations made at the European Southern Observatory and the Cerro Tololo Inter-American Observatory. This publication makes use of data products from the Two Micron All Sky Survey, which is a joint project of the University of Massachusetts and the Infrared Processing and Analysis Center/California Institute of Technology, funded by the National Aeronautics and Space Administration and the National Science Foundation. This publication makes use of the ESO/ST-ECF Science Archive Facility.

\section{References}

Alcalá, J. M., Covino, E., Torres, G., et al. 2000, A\&A, 353, 186 Arellano Ferro, A., \& Giridhar, S. 2003, A\&A, 408, L29

Baraffe, I., Chabrier, G., Allard, F., \& Hauschildt, P. H. 1998, A\&A, 337,403

Batten, A. H., Fletcher, J. M., \& MacCarthy, D. G. 1989, Publ. DAO, 17

Covino, E., Alcalá, J. M., Allain, S., et al. 1997, A\&A, 328, 187
Covino, E., Melo, C. H. F., Alcala, J. M., et al. 2001, A\&A, 375, 130

Cutri, R. M., Skrutskie, M. F., Van Dyk, S., et al. 2003, 2MASS All Sky Data Release,

http://www.ipac.caltech.edu/2mass/releases/allsky/

Delfosse, X., Forveille, T., Perrier, C., \& Mayor, M. 1998, A\&A, 331, 581

de Jager, C., \& Nieuwenhuijzen, H. 1987, A\&A, 177, 217

Duquennoy, A., \& Mayor, M. 1991, A\&A, 248, 485

Edwards, S., Hartigan, P., Ghandour, L., \& Andrulis, C. 1994, AJ, 108, 1056

Gahm 1999, A\&A, 352, L95

Ghez, A., Neugebauer, G., \& Matthews, K. 1993, AJ, 106, 2005

Kaufer, A., Stahl, O., Tubbesing, S., et al. 1999, The ESO Messenger, 95, 8

Kiseleva, L. G., Eggelton, P. P., \& Mikkola, S. 1998, MNRAS, 200, 292

Leinert, C., Henry, T., Glindemann, A., \& McCarthy, D. W. 1997, A\&A, 325, 159

Martin, E. 1997, A\&A, 321, 492

Mayor, M., \& Mazeh, T. 1987, A\&A, 171, 157

Melo, C. H. F., Covino, E., Alcalá, J. M., \& Torres, G. 2001, A\&A, 738,898

Melo, C. H. F., Pasquini, L., \& De Meideros, J. R. 2001, A\&A, 375, 851

Melo, C. F. H. 2003, A\&A, 410, 269

Mermilliod, J. C., \& Mayor, M. 1999, A\&A, 353, 479

Mermilliod, J. C., Rosvick, J. M., Duquennoy, A., \& Mayor, M. 1992, A\&A, 265, 513

Montes, D., Martín, E. L., Fernandez-Figueroa, M. J., Cornide, M., \& de Castro, E. 1997, A\&AS, 123, 473

Mora, A., Merin, B., Solano, E., et al. 2001, A\&A, 378, 116

Oudmaijer, R. D., van der Veen, W. E. C. J., Waters, L. B. F. M., et al. 1992, A\&AS, 96, 625

Prato, L., Greene, T. P., \& Simon, M. 2003, ApJ, 584, 853

Probst, R. G., Montane, A., Warner, M., et al. 2003, Proc. SPIE, 4841, 411

Reipurth, B., Lindgren, H., Mayor, M., Mermilliod, J.-C., \& Cramer, N. 2002, AJ, 124, 2813

Sterzik, M. F., Durisen, R. H., \& Zinnecker, H. 2003, A\&A, 411, 91

Sterzik, M. F., \& Tokovinin, A. A. 2002, A\&A, 384, 1030

Sylvester, R. J., \& Skinner, C. J. 1996, MNRAS, 283, 475

Tokovinin, A. A. 1997, A\&AS, 124, 75

Tokovinin, A. A., \& Smekhov, M. G. 2002, A\&A, 382, 118

Torres, G., Neuhäuser, R., \& Günther, E. 2002, AJ, 123, 1701

Torres, G. 2004, ApJ, 127, 1187

Wichmann, R., Torres, G., Melo, C. H. F., et al. 2000, A\&A, 359, 181

Zuckerman, B., Forveille, T., \& Kastner, J. H. 1995, Nature, 373, 494 